\title{
MOLECULAR PHYLOGENY OF THE BALLISTOCONIDIUM- FORMING ANAMORPHIC YEAST GENUS BULLERA AND RELATED TAXA BASED ON SMALL SUBUNIT RIBOSOMAL DNA SEQUENCES
}

\author{
SUNG-OUI SUH, ${ }^{\dagger}$ MASAKO TAKASHIMA, ${ }^{1}$ MAKIKO HAMAMOTO, ${ }^{1}$ \\ AND TAKASHI NAKASE ${ }^{1, *}$ \\ Deep-Sea Microorganisms Research Group, Japan Marine Science and Technology Center, \\ Yokosuka 237, Japan \\ ${ }^{1}$ Japan Collection of Microorganisms, The Institute of Physical and Chemical Research (RIKEN), \\ Wako 351-01, Japan
}

(Received June 18, 1996; Accepted November 10, 1996)

The sequence of the small subunit ribosomal coding gene (SSU rDNA) was determined for the type strains of seven species of the genus Bullera and two species which are now regarded as synonyms of species in the genera Bullera and Udeniomyces. The phylogenetic trees for these yeasts were constructed by neighbor-joining and maximum likelihood methods, including supposedly related yeasts and filamentous fungi whose SSU rDNA sequences were already known. Fifteen presently recognized species of Bullera were divided into two phylogenetic groups. Bullera mrakii, B. huiaensis, B. sinensis, B. oryzae, B. coprosmaensis, B. crocea, $B$. armeniaca, and $B$. variabilis constitute one cluster. In this cluster, the topologies of $B$. variabilis and orange-colored species, $B$. crocea and $B$. armeniaca, are different according to the neighbor-joining and maximum likelihood trees. Bullera pseudoalba, B. alba, B. unica, B. hannae, B. globispora, $B$. miyagiana, and $B$. dendrophila constitute another cluster. In this cluster, $B$. pseudoalba, $B$. alba, $B$. unica, and $B$. hannae constitute a branch but $B$. globispora, $B$. miyagiana, and $B$. dendrophila are located at positions far from one another although the former two species constitute a cluster in the maximum likelihood tree. Among the above species, B. dendrophila constitutes a cluster with Tsuchiyaea wingfieldii and Filobasidiella neoformans. The neotype strain of $B$. grandispora, which is now regarded a synonym of Udeniomyces piricola, is located close to the position of type strain $U$. piricola.

\footnotetext{
* Address reprint requests to: Dr. Takashi Nakase, Japan Collection of Microorganisms, The Institute of Physical and Chemical Research (RIKEN), 2-1 Hirosawa, Wako 351-01, Japan.

${ }^{\dagger}$ Present address: Department of Plant Biology, Louisiana State University, Baton Rouge, Louisiana 70803, U.S.A.
} 
Since the establishment of the genus Bullera by Derx (3), this genus has been differentiated from the genus Sporobolomyces by pale colored colonies and symmetrical ( = rotationally symmetrical) ballistospores (ballistoconidia), which basically have globose bodies. Phaff and do Carmo-Sousa (14) emended the definition of the genus Sporobolomyces to include $S$. singularis, which produces pale colored colonies like Bullera species. Stadelmann (17) emended the definition of the genus Bullera to include $B$. piricola, which produces asymmetrical as well as symmetrical ballistospores. In 'The Yeasts, a Taxonomic Study (3rd ed.),' published in 1984, Rodrigues de Miranda (15) and Fell and Statzell Tallman (5) stated that Bullera species usually produced rotationally symmetrical ballistospores and cream colored to slightly yellowish or brownish colonies, whereas Sporobolomyces species usually produced bilaterally symmetrical ballistospores and pink, red, and orange colonies. The former author transferred S. singularis to the genus Bullera based on the observation of pale colored colonies even though it produced bilaterally symmetrical ballistospores. Furthermore, Buhagiar et al. (2) and Johri and Bandoni (9) described several Bullera species producing deep-orange colonies. Therefore, the distinction between the genera Bullera and Sporobolomyces became confusing.

In 1983, Weijman and Rodrigues de Miranda (30) pointed out the significance of the presence or absence of xylose in whole-cell hydrolysates in the taxonomy of species of these genera; xylose is present in the majority of the genus Bullera, but not in the genus Sporobolomyces. However, their data were not clear-cut and some discrepancies were found at that time. Later, in 1988, Suzuki and Nakase (23) analyzed monosaccharide composition of whole-cell hydrolysates of species of Bullera and Sporobolomyces, including species of Sporidiobolus, which is considered to be a teleomorph of Sporobolomyces. They found that these genera were clearly separated according to the presence or absence of cellular xylose. They suggested the transfer of xylose-lacking species, Bullera salicina and B. tsugae, to Sporobolomyces as well as the transfer of xylose-containing species, Sporobolomyces puniceus, to Bullera.

In 1992, Nakase and Takematsu (12) proposed a new genus Udeniomyces for three Bullera species, B. piricola, B. megalospora, and B. punicea, based on the analysis of the partial sequences of small subunit ribosomal RNA (SSU rRNA) and the production of large, asymmetrical ballistospores. The genus Bullera now comprises species with xylose in their cell hydrolysates, which produce rotationally symmetrical (basically globose but not elongate) ballistoconidia.

Nakase et al. (13) studied the molecular phylogeny of ballistosporous yeasts based on the partial sequence of SSU rRNA and found that species of xylosecontaining basidiomycetous yeasts constitute a cluster that is different from that comprising xylose-lacking species. Species of Bullera are located in the cluster together with species of other xylose-containing basidiomycetous yeast genera. Suh and Nakase (18) analyzed the complete sequence of SSU rRNA coding gene (SSU rDNA) of several species of Bullera and Udeniomyces and confirmed the separation of Udeniomyces from Bullera. They found that species of Bullera constituted a 
cluster mixed with Filobasidiella neoformans, Tremella globospora, and Trichosporon cutaneum. This result clearly suggests the heterogeneity of the genus Bullera as discussed in that paper (18). Moreover, recently five new species were proposed in Bullera based on the strains isolated from various plant materials collected in New Zealand (8).

We studied sequences of SSU rDNA of all known species of Bullera, including five recently described species in order to elucidate the phylogenetic relationships among the species of Bullera and their relationships between Bullera and other basidiomycetous yeast genera.

\section{MATERIALS AND METHODS}

Strains employed. The following yeast strains were employed for sequencing of SSU rDNA: B. armeniaca JCM $2955^{\mathrm{T}}$, B. coprosmaensis $\mathrm{JCM} 8936^{\mathrm{T}}$, B. derxii (=B. sinensis) $\mathrm{JCM} 5280^{\mathrm{T}}$, B. grandispora (=Udeniomyces piricola) $\mathrm{JCM} 8249^{\mathrm{NT}}$, B. hannae $\mathrm{JCM} 8937^{\mathrm{T}}$, B. huiaensis $\mathrm{JCM} 8933^{\mathrm{T}}$, B. mrakii $\mathrm{JCM} 8934^{\mathrm{T}}$, B. sinensis JCM $6253^{\mathrm{T}}$, and $B$. unica $\mathrm{JCM} 8932^{\mathrm{T}}$. Strains with superscript $\mathrm{T}$ were derived from the type, and that with NT was indicated as neotype.

PCR and sequencing of SSU rDNA. DNA was obtained from cells broken by sonication. After purification of DNA, the PCR and sequencing of SSU rDNA were carried out according to Suh et al. (21). The following primers were employed for sequencing: 5'-TGGAATTACCGCGGCTGCTGGCACC-3' (approximate position 585-561 in Saccharomyces cerevisiae), 5'-CCGTCAATTCCTTTAAGTTTCAGCC-3' (position 1,147-1,123), 5'-TCTGGGCCGCACGCGCGCTACACTG-3' (position 1,448-1,472), and 5'-GACGGGCGGTGTGTACAAAGGGCAG-3' (position 1,642-1,618). The nucleotide sequence data reported in this paper will appear in the DDBJ, EMBL, and Gen Bank nucleotide sequence databases with the following accession numbers: D78323 (B. armeniaca), D78324 (B. grandispora), D78325 (B. mrakii), D78326 (B. coprosmaensis), D 78327 (B. hannae), D78328 (B. sinensis), D78329 (B. derxii), D78330 (B. unica), and D 78331 (B. huiaensis).

Phylogenetic analysis. The SSU rDNA sequences were aligned by using the multiple alignment program CLUSTAL W (29) with the sequences of other known sequence data from the nucleotide sequence libraries in EMBL, Gen Bank, and DDBJ. For the analysis using the neighbor-joining method (16), distances between the sequences were calculated using Kimura's two parameter model (10). Sites where gaps existed in any of the sequences were excluded. Bootstrap analysis (6) was performed from 1,000 random resampling. The DNAML program in the PHYLIP 3.5c package (7) was used for maximum likelihood analysis with a transition/transversion ratio of PHYLIP of 2.000000 . 


\section{RESULTS AND DISCUSSION}

About 1,750 bases of SSU rDNA were determined for each strain. For 62 yeasts and filamentous fungi, the sequence data were aligned including 53 already published data, and the phylogenetic trees were constructed by neighbor-joining (Fig. 1) and maximum likelihood methods (Fig. 2). The ascomycetous yeasts, Saccharomyces cerevisiae, Kluyveromyces lactis, and Candida albicans, and the filamentous ascomycete Neurospora crassa, were employed as an outgroup.

As the result of phylogenetic analysis, the basidiomycetes were divided into three major clusters as reported in several recent papers $(4,18-22,24-28)$. Firstly, the species of the smut fungi (Ustilaginales), Ustilago and Tilletia, were diverged as cluster 1. The remaining species of basidiomycetes were separated into two clusters, 2 and 3, which correlated with the known presence or absence of xylose in the cells according to available data (Fig. 1).

All of the species of xylose-containing yeasts were located in cluster 3 , in which two subclusters, $3 \mathrm{a}$ and $3 \mathrm{~b}$, are recognized. The basidiomycetous yeast genera Phaffia, Mrakia, Udeniomyces, and Cystofilobasidium were located in subcluster 3a. They constituted a good branch in respective trees (Figs. 1 and 2), and were considered to be monophyletic. Some species of mushrooms in Homobasidiomycetes and Heterobasidiomycetes were also located in subcluster 3a.

Species of Udeniomyces resemble Sporobolomyces in their ballistoconidium morphology and resemble Bullera in their physiological and biochemical characteristics. Therefore, $U$. puniceus, the longest known species of this genus, which was once included in Sporobolomyces, transferred to Bullera, and was then included in Udeniomyces, which was proposed based on the partial sequence of SSU rRNA and the morphology of ballistospores (ballistoconidia) (12). Species of Udeniomyces utilize nitrate and produce distinct scars during conidiogenesis different from those in Bullera species (1). Later, the separation of Udeniomyces from Bullera was supported by complete sequences of SSU rDNA (18).

A strain labeled Bullera grandispora was located at a position close to that of the type strain of Udeniomyces piricola. Bullera grandispora was described by Derx (3) based on a strain isolated from a leaf of chestnut infected with mildew. Unfortunately, the culture of this strain is not maintained at any place in the world. An isolate assigned to this species was sent to Centraalbureau voor Schimmelcultures (CBS) by R. J. Bandoni and listed in the 30th edition of CBS List of Cultures (1983) as B. grandispora. Nakase and Suzuki (11) found that this strain was identical to $B$. piricola based on the morphology, physiology, and electrophoretic comparison of ten enzymes, and regarded $B$. grandispora as a nomen dubium because the original description of this species given by Derx (3) is too short to unequivocally characterize the species. The present study also supported the inclusion of the strain in $U$. piricola.

The species of Bullera whose SSU rDNAs were sequenced in the present study 


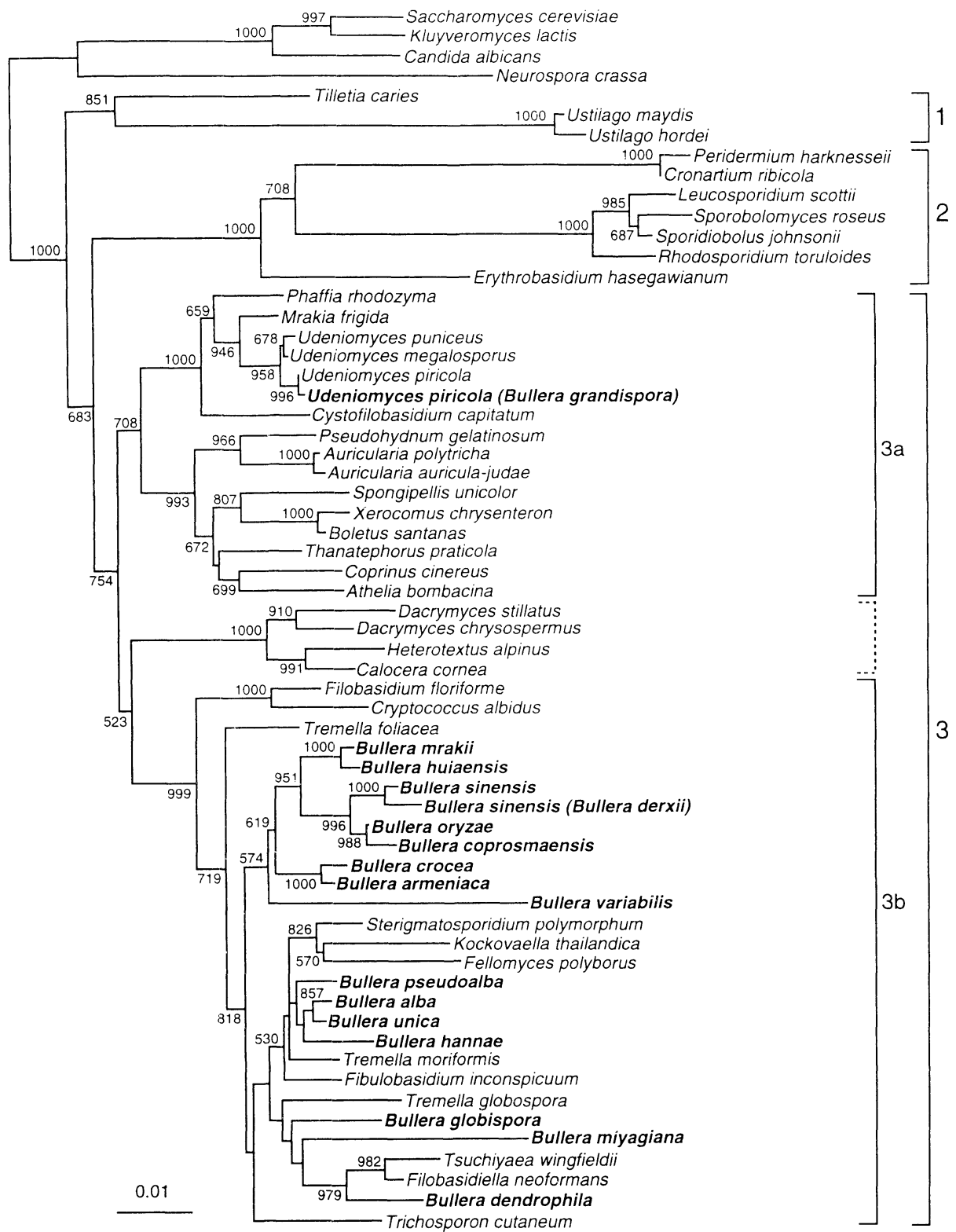

Fig. 1. Phylogenetic tree of basidiomycetous yeasts and fungi including all the known species of the genus Bullera based on the SSU rDNA sequence.

The tree was constructed by the neighbor-joining method. Each number indicates the bootstrap confidence level derived from 1,000 resampling (the frequency less than $50 \%$ was not indicated). The distance corresponding to one base change per hundred nucleotide positions is indicated by a bar. 


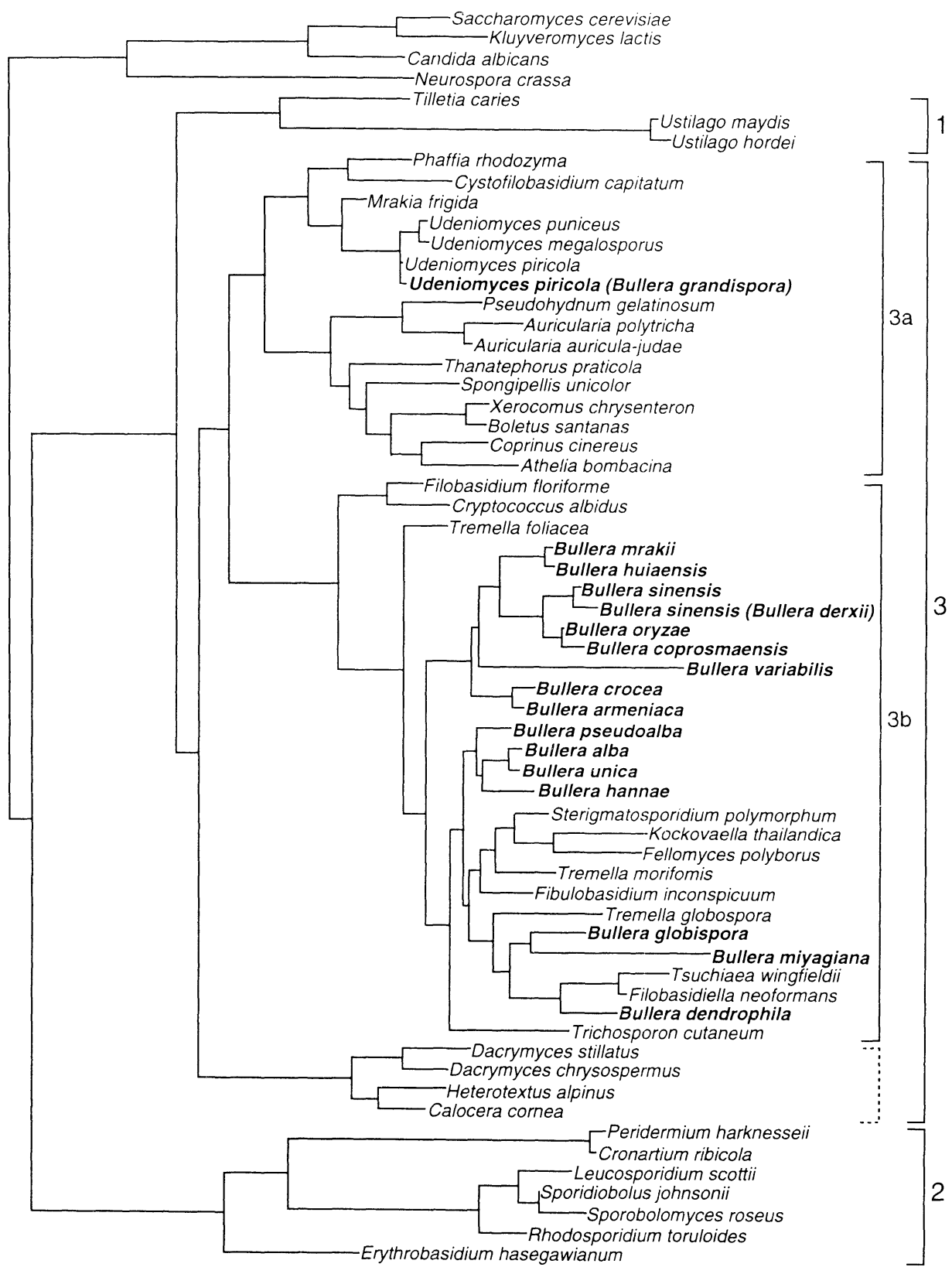

Fig. 2. Phylogenetic tree of basidiomycetous yeasts and fungi including all the known species of the genus Bullera based on the SSU rDNA sequence.

The tree was constructed by the maximum likelihood method. 
were located in subcluster $3 \mathrm{~b}$, and contained all of the known species of Bullera (Figs. 1 and 2). Yeast species in the genera Filobasidium, Cryptococcus, Sterigmatosporidium, Kockovaella, Fellomyces, Tsuchiyaea, Filobasidiella, and Trichosporon in addition to species of Tremella and Fibulobasidium (Tremellales) were also located in the subcluster. Several species of Dacrymycetales (Homobasidiomycetes) are clustered with subcluster $3 \mathrm{~b}$ in the neighbor-joining tree (Fig. 1), but were located outside the subclusters $3 \mathrm{a}$ and $3 \mathrm{~b}$ in the maximum likelihood tree (Fig. 2). Swann and Taylor reported that the species of Dacrymycetales were clustered with those of Auriculariales (subcluster $3 \mathrm{a}$, in this paper) $(25,26)$. Apparently, further sequencing studies are required to clarify the phylogenetic positions of fungi of Dacrymycetales.

Species of Bullera occurred in several branches of subcluster $3 \mathrm{~b}$, together with yeasts of other basidiomycetous genera. Bullera mrakii, $B$. huiaensis, $B$. sinensis, $B$. derxii, B. oryzae, B. coprosmaensis, B. crocea, B. armeniaca, and $B$. variabilis constituted a branch of their own. It is not certain whether this group of Bullera species is monophyletic because the SSU rDNA of many xylose-containing yeast species such as those of Cryptococcus have not been analyzed yet. Bullera derxii constituted a branch with the type strain of $B$. sinensis. The similarity value of the sequence of these two strains is $99.1 \%$ and does not contradict the conspecificity of these strains.

The remaining seven species, including the type species $B$. alba, were located at several branches in subcluster 3 b (Figs. 1 and 2). Bullera pseudoalba, B. alba, $B$. unica, and $B$. hannae constituted a branch of their own, and were considered to be closely related. On the contrary, B. globispora, B. miyagiana, and B. dendrophila were scattered among subcluster $3 \mathrm{~b}$, and mixed with species of the genera Sterigmatosporidium, Kockovaella, Fellomyces, Fibulobasidium, Tsuchiyaea, Filobasidiella, Trichosporon, and Tremella (Tremellales). Filobasidium floriforme, Cryptococcus albidus, and Tremella foliacea were located in subcluster $3 \mathrm{~b}$ but outside the cluster where Bullera species were located (Figs. 1 and 2).

As discussed above, the topologies shown in Figs. 1 and 2 clearly suggested that the present separation of basidiomycetous yeast genera does not necessarily reflect the phylogenetic relationship of basidiomycetous yeasts. In short, the genus Bullera seems to be not well-defined and is comprised of species with different phylogenetic origins. Further sequencing study including all basidiomycetous yeast species is essential for a correct understanding of the phylogeny of species of Bullera.

\section{REFERENCES}

1) Boekhout, T., A revision of ballistoconidia-forming yeasts and fungi. Stud. Mycol., 33, 1-194 (1991).

2) Buhagiar, R. W. M., Yarrow, D., and Barnett, J. A., Bullera crocea and Bullera armeniaca, two new yeasts from fruit and vegetables. J. Gen. Microbiol., 129, 3149-3155 (1983). 
3) Derx, H. G., Étude sur les Sporobolomycétes. Ann. Mycol., 28, 1-23 (1930).

4) Fell, J. W., Boekhout, T., and Freshwater, D. W., The role of nucleotide sequence analysis in the systematics of the yeast genera Cryptococcus and Rhodotorula. Stud. Mycol., 38, 129-146 (1995).

5) Fell, J. W. and Statzel Tallman, A., Genus Sporobolomyces Kluyver et van Niel. In The Yeasts, a Taxonomic Study, 3rd. ed., ed. by Kreger-van Rij, N. J. W., Elsevier Sci. Publ., Amsterdam (1984), p. 911-929.

6) Felsenstein, J., Confidence limits on phylogenies: An approach using the bootstrap. Evolution, 39, 783-791 (1985).

7) Felsenstein, J., PHYLIP (Phylogeny Inference Package) version 3.5c., distributed by the author, Department of Genetics, University of Washington, Seattle.

8) Hamamoto, M. and Nakase, T., Ballistosporous yeasts found on the surface of plant materials collected in New Zealand. Antonie van Leeuwenhoek, 69, 279--291 (1996).

9) Johri, B. N. and Bandoni, R. J., Taxonomy of the sporobolomycetous genus Bullera. In Taxonomy of Fungi. Proc. Int. Symp. Taxonomy of Fungi, 1973, Part 2, Univ. Madras, p. 536547 (1984).

10) Kimura, M., A simple method for estimating evolutionary rate of base substitutions through comparative studies of nucleotide sequences. J. Mol. Evol., 16, 111-120 (1980).

11) Nakase, T. and Suzuki, M., Bullera megalospora, a new species of yeast forming large ballistospores isolated from dead leaves of Oryza sativa, Miscanthus sinensis and Sasa sp. in Japan. J. Gen. Appl. Microbiol., 32, 225-240 (1986).

12) Nakase, T. and Takematsu, A., Udeniomyces, a new ballistosporous anamorphic yeast genus in the Cryptococcaceae proposed for three Bullera species which produce large bilaterally symmetrical ballistospores. FEMS Microbiol. Lett., 100, 497-502 (1992).

13) Nakase, T., Takematsu, A., and Yamada, Y., Molecular approaches to the taxonomy of ballistosporous yeasts based on the analysis of the partial nucleotide sequences of $18 \mathrm{~S}$ ribosomal ribonucleic acids. J. Gen. Appl. Microbiol., 39, 107-134 (1993).

14) Phaff, H. J. and do Carmo-Sousa, L., Four new species of yeast isolated from insect frass in bark of Tsuga heterophyla (Raf.) Sargent. Antonie van Leeuwenhoek, 28, 193-207 (1962).

15) Rodrigues de Miranda, L., Genus Bullera. In The Yeasts, a Taxonomic Study, 3rd ed., ed. by Kreger-van Rij, N. J. W., Elsevier Sci. Publ., Amsterdam (1984), p. 577-584.

16) Saitou, N. and Nei, M., The neighbour-joining method: A new method for reconstructing phylogenetic trees. Mol. Biol. Evol., 4, 406-425 (1987).

17) Stadelmann, F., A new species of the genus Bullera Derx. Antonie van Leeuwenhoek, 41, 575-582 (1975).

18) Suh, S.-O. and Nakase, T., Phylogenetic analysis of the ballistosporous anamorphic genera Udeniomyces and Bullera, and related basidiomycetous yeasts based on 18S rDNA sequences. Microbiology, 141, 901-906 (1995).

19) Suh, S.-O. and Sugiyama, J., Phylogeny among the basidiomycetous yeasts inferred from small subunit ribosomal DNA sequence. J. Gen. Microbiol., 139, 1595-1598 (1993).

20) Suh, S.-O. and Sugiyama, J., Phylogenetic placement of the basidiomycetous yeasts Kondoa malvinella and Rhodosporidium dacryoidum, and the anamorphic yeast Sympodiomycopsis paphiopedili by means of 18S rRNA gene analysis. Mycoscience, 35, 367-375 (1994).

21) Suh, S.-O., Takashima, M., and Nakase, T., Phylogenetic study of the anamorphic basidiomycetous yeasts Rhodotorula lactosa and $R$. minuta, and related taxa based on $18 \mathrm{~S}$ rDNA sequences. $J$. Gen. Appl. Microbiol., 42, 1-6 (1996).

22) Suh, S.-O., Takematsu, A., Takashima, M., and Nakase, T., Molecular phylogenetic study on the stalked conidium-forming yeasts and related basidiomycetous yeast taxa based on 18S rDNA sequences. Microbiol. Cult. Coll., 12, 79-86 (1996).

23) Suzuki, M. and Nakase, T., The distribution of xylose in the cells of ballistosporous yeastsApplication of high performance liquid chromatography without derivatization to the analysis of xylose in whole cell hydrolysates. J. Gen. Appl. Microbiol., 34, 95-103 (1988). 
24) Swann, E. C. and Taylor, J. W., Higher taxa of basidiomycetes: An 18S rRNA gene perspective. Mycologia, 85, 923-936 (1993).

25) Swann, E. C. and Taylor, J. W., Phylogenetic perspectives on basidiomycete systematics: Evidence from the 18S rRNA gene. Can. J. Bot., 73 (Suppl. 1), S862-S868 (1995).

26) Swann, E. C. and Taylor, J. W., Phylogenetic diversity of yeast-producing basidiomycetes. Mycol. Res., 99, 1205-1210 (1995).

27) Takashima, M., Suh, S.-O., and Nakase, T., Phylogenetic relationships among species of the genus Bensingtonia and related taxa based on the small subunit ribosomal DNA sequences. J. Gen. Appl. Microbiol., 41, 131-141 (1995).

28) Takashima, M., Suh, S.-O., and Nakase, T., Bensingtonia musae sp. nov. isolated from a dead leaf of Musa paradisiaca and its phylogenetic relationship among basidiomycetous yeasts. J. Gen. Appl. Microbiol., 41, 143-151 (1995).

29) Thompson, J. D., Higgins, D. G., and Gibson, T. J., CLUSTAL W: Improving the sensitivity of progressive multiple sequence alignment through sequence weighting, position-specific gap penalties and weight matrix choice. Nucleic Acid Res., 22, 4673-4680 (1994).

30) Weijman, A. C. M. and Rodrigues de Miranda, L., Xylose distribution within and taxonomy of the genera Bullera and Sporobolomyces. Antonie van Leeuwenhoek, 49, 559-562 (1983). 